\title{
Design of the Low-energy DC Gun for Field-emission Cathode Investigation
}

\author{
Anusorn Lueangaramwong \\ Northern Illinois University \\ DeKalb IL, USA \\ anusorn@nicadd.niu.edu
}

\author{
Osama Mohsen \\ Northern Illinois University \\ DeKalb IL, USA
}

\author{
Philippe Piot \\ Northern Illinois University \& \\ Fermi National Accelerator Laboratory \\ DeKalb IL, USA
}

\begin{abstract}
A DC electron source test-stand to explore fieldemission from various cathodes has been designed. The design also includes a provision to allow for laser-triggered field emission using a femtosecond laser source. The gun is instrumented to measure the beam transverse distribution, the transverse emittance, and $I-V$ characteristic curves. The electrostatic design of the gun and multi-particle simulations of the beamline are presented and planned experiment discussed.
\end{abstract}

Index Terms - field emission, DC electron sources, emittance

\section{INTRODUCTION}

Field emission - the macroscopic manifestation of quantum tunneling - is a promising electron-emission mechanism which has been widely used in vacuum electronic and display technologies. The emission occurs as a strong electric field $\mathcal{O}(\mathrm{GV} / \mathrm{m})$ is applied at the surface a of material. The theory of the field emission is well established [1], [2] and the instantaneous current density at a location on the emitter surface is described by the Fowler-Nordheim's (F-N) law [1].

A single-tip field-emitter (FE) cathode emits electrons at a sharp tip edge with nanoscale curvature and generates beams with corresponding small beam size as well as low transverse emittances [3]. Furthermore, a field-emissionarray (FEA) cathode - FEs arranged as large arrays produces higher average currents [4] at the expense of larger emittance. The electron sources with the FE cathodes can be anticipated to provide extremely high brightness corresponding to the quantum-limited emittance $\varepsilon_{q} \equiv N \lambda_{c}^{3} / 2$, where $N$ is the number of electrons and $\lambda_{c}$ is the Compton wavelength. Additionally, an array of regularly-organized tips provides a source of transversely-segmented electron beamlets which could be subsequently manipulated for advanced applications [5], [6].

The DC gun test-stand discussed in this paper was designed as a versatile system to ultimately support the investigation of low-emittance beams from FE cathodes. In its first step it will be used to characterize the emittance and investigate simple beam manipulations associated with beams from FEA cathodes (either in the high-current or patterned beams). One of the main differences among the various applications is the DC extraction-electrode system. While the formation of ultracold beam from a single emitter will most likely employ a micro-fabricated extraction anode or one that follows an approach similar to Ref. [7]. The beam generation from an FEA will require a different design.

\section{OVERVIEW}

The DC gun test-stand under construction is diagrammed in Fig. 1. In brief an FE (or FEA) cathode is grounded an located in close proximity to a anode at a high-voltage potential. After extraction the field-emitted beam is accelerated to up to $25 \mathrm{kV}$. The purpose of this DC gun test-stand is to experimentally characterize various FE cathodes in a DC gap, where a high electrostatic field is applied. The current is measured between the cathode and ground. The beamline also incorporates a set of printed-circuit-board quadrupole magnets [8]. The latter quadrupole magnets will be used to image the beam and check some of the beam-matching conditions derived in Ref. [6] while also enabling the transverse-emittance measurement using the quadrupole-scan method. Ultimately, the system will support the investigation and characterization $(I-V$ curve, emittance, lifetime) of an array of silicon-based FE cathodes (e.g. with different emitters geometry, material and doping concentration, vacuum level, etc).

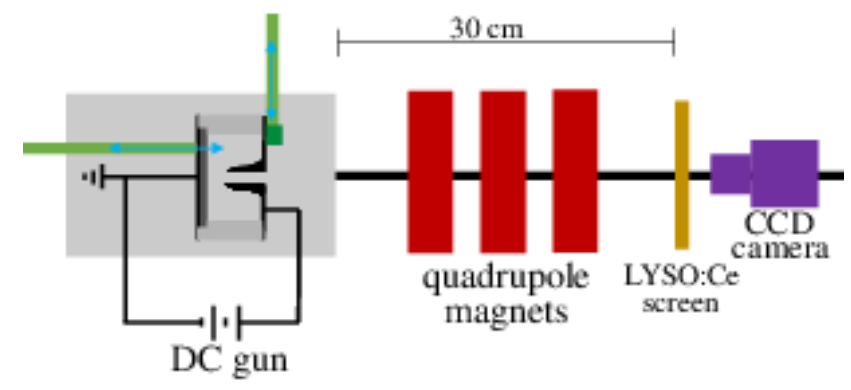

Fig. 1. Overview of the DC-gun beamline for field emission cathode characterization.

\section{EXTRACTION ELECTRODES}

The extraction-electrode geometry was simulated with WARP [9]. One of the main challenges regards the use of Silicon-based FE cathodes (which are fabricated on a flat silicon wafer) together with the need to "shield" the cathodewafer irregular cut prevent us from using a Pierce-cathode geometry. Therefore only the anode-assembly adapts to such a configuration. An optimized configuration appears in Fig. 2.

This manuscript has been authored by Fermi Research Alliance, LLC under Contract No. DE-AC02-07CH11359 with the U.S. Department of Energy, Office of Science, Office of High Energy Physics. 
In our configuration, the cathode is grounded so that a picoammeter measures the current at zero potential. The anodes are subjected to a high voltage of up to +5 and $+30 \mathrm{kV}$. The cathode is mounted on a linear motion stage to vary the cathodeanode gap; see Fig. 3. The cathode plug is electrically isolated from the stage using an insulating Techtron ${ }^{\circledR}$ frame. The anode-electrode assembly is mounted on another manuallycontrollable linear stage that enables its vertical alignment under vacuum (the system is horizontally aligned during the installation).
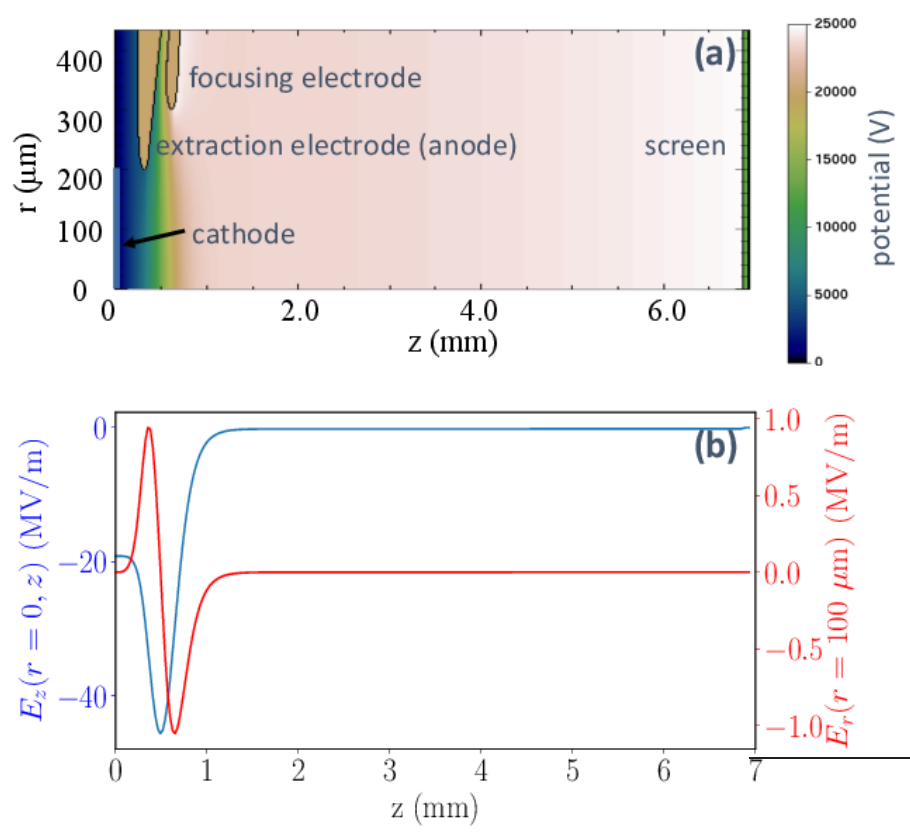

Fig. 2. Computational domain for solving the electrostatic problem (a) and axial (blue) and off-axis transverse (red trace) electric field (b) along the longitudinal coordinate $z$. The value $z=0$ correspond to the location of the cathode and the false-color map in (a) represents the scalar potential.

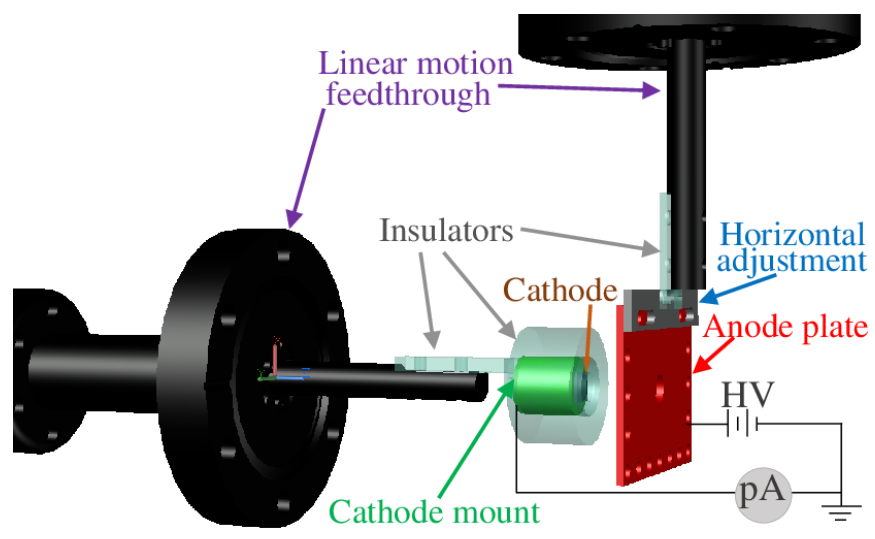

Fig. 3. Close up view of the cathode/anode assembly.

The performance of the proposed setup was optimized via numerical simulation in WARP using the cylindrical-symmetric electrostatic solver. Figure 2(a) shows the computational $r-z$ domain. The domain extends over $r \times z=1.8 \times 6.5 \mathrm{~mm}^{2}$ and is discretized with a $129 \times 258$ uniform grid. The axial boundaries are a grounded conducting plate (at $z=0$ ) including a cathode with radius $200 \mu \mathrm{m}$ and a screen (at $z=7 \mathrm{~mm}$ ) subjected the same potential as the focusing electrode $V_{f}$. The anode is set to a potential $V_{e}$. The anode and focusing electrode aperture radii are respectively 200 and $300 \mu \mathrm{m}$ and both have a Pierce angle of $59^{\circ}$. The electrostatic simulations presented in Fig. 2(a) correspond to the case $V_{e}=5 \mathrm{kV}$ and $V_{f}=25 \mathrm{kV}$.

An apertured anode generates radial field $E_{r}(r, z)=$ $\frac{d E_{z}(z, r)}{d z}$ where $(r, z)$ represents the cylindrical coordinates associated to the axially symmetric system. Figure 2(b) displays the axial $E_{z}(r=0, z)$ and radial $E_{r}(r=100 \mu \mathrm{m}, z)$ electric field as a function of the axial distance. Under a nominal cathode-anode spacing of $252 \mu \mathrm{m}$, the peak electric field on the cathode surface is $\left|E_{z}(0,0)\right| \simeq 20 \mathrm{MV} / \mathrm{m}$. When compared with a setup with a flat anode (i.e. without a center hole), the present cathode-anode geometry yields lower electric fields at the cathode. Another point of concern regards the radial field in the close vicinity of the cathode surface which corresponds to a radially-dependent axial electric field. Such a radial dependence could affect the field-emission uniformity across the cathode surface as shown in Fig. 4(a). The latter figure indicates that the relative field variation across the cathode ( $r \in[0,200] \mu \mathrm{m})$ is approximately $\pm 1 \%$. The corresponding current dependence on $r$ appears in Fig. 4(b) and is negligible across the cathode surface.

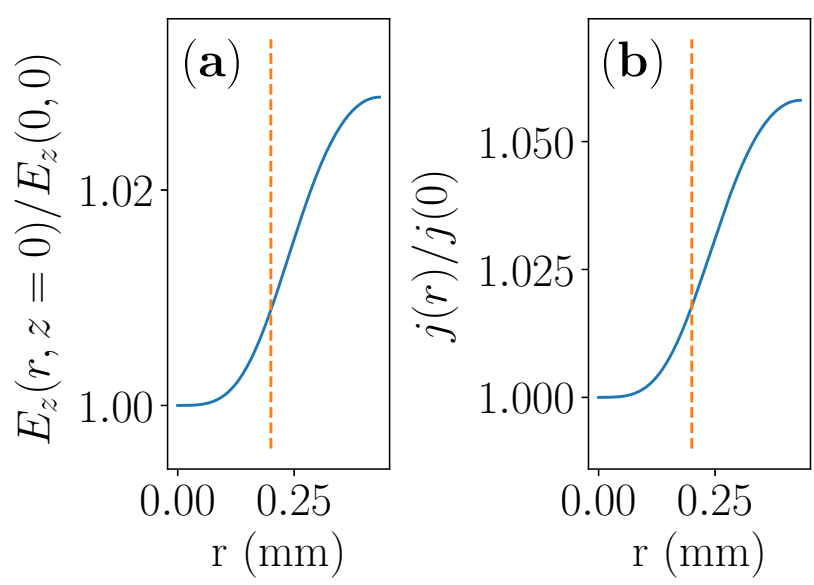

Fig. 4. Radial dependence of the axial electric field on the cathode surface normalized to its on-axis value (a) and corresponding current density $j$ normalized to its on axis value $j(r=0)$. The dashed vertical lines indicate the outer radius of the cathode.

\section{BEAM-DYNAMICS SIMULATIONS}

The beam dynamics in the DC gun was explored with the WARP program. The electrostatic field is first solved across the computational domain and then the emission of electron modeled using the same solver. The cathode located at $z=0 \mathrm{~m}$ is modeled as a field-emission source following the model described in Ref. [6]. To avoid modeling the 
specific nanostructure of the cathode, for sake of speed and memory demand, we introduce a field-enhancement factor $\beta_{e} \equiv E_{\text {surf }} / E_{m a c}$ as the ratio of the macroscopic field $E_{\text {mac }}$ associated with the cathode-anode geometry with an enhanced surface field $E_{\text {surf }}$ owing to the emitting surface microscopic geometry. Figure 5 shows field emission in the setup, while electrostatic lenses are applied. The result shows the possibility to both trigger field emission and focuses the beam while having difficulties from an anode with a hole to allow emitted electrons to escape to the beamline.
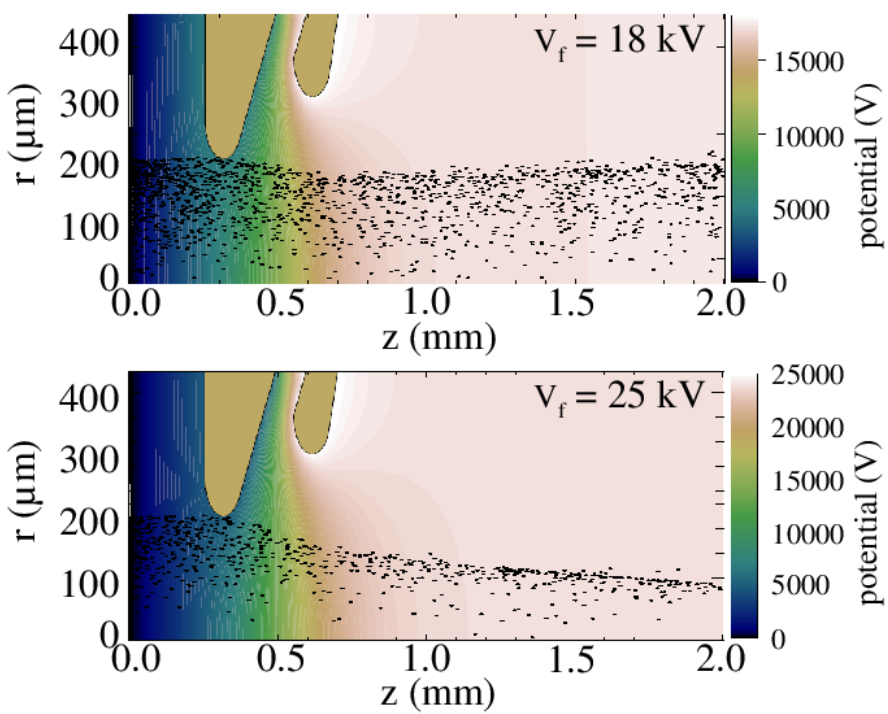

Fig. 5. Field emission at $\mathrm{t}=0.4 \mathrm{~ns}$ after emission starts. The field emission is triggered on a flat surface with field enhancement $\beta_{e}$ of 157 at $\mathrm{z}=0 \mathrm{~mm}$. A spacing between the cathode and the first anode is $0.252 \mathrm{~mm}$. The anode, focusing electrode and conductive screen at $\mathrm{z}=6.93 \mathrm{~mm}$ are applied with voltage of $V_{e}=5 \mathrm{kV}, V_{f}=18 \mathrm{kV}$ (top), $25 \mathrm{kV}$ (bottom) and $V_{f}=18 \mathrm{kV}$ (top), $25 \mathrm{kV}$ (bottom), respectively. The outer surface of the first anode and the inner surface of focusing electrode make an angle of 59 degree with $+z$ axis, while having hole radius of $0.2 \mathrm{~mm}$ and $0.3 \mathrm{~mm}$, respectively. A spacing between the anode and focusing electrode is $0.240 \mathrm{~mm}$. Black dots represent microparticles. Beam current recorded at the cathode is approximately 0.8 (top) and 0.4 (bottom) $\mu \mathrm{A}$.

As indicated in Fig. 2(b) the apertured anode does not only reduce the field amplitude on the cathode surface but also defocuses the beam owing to the transverse fields generated by the edge of the aperture. The focusing anode provides a focusing force to control the beam size before further transport through other focusing elements such as a solenoid or a set of quadrupole magnets. The geometries of two anodes illustrated in Fig. 5 provide a net focusing force which depends on the relative potential difference $V_{f}-V_{e}$. The latter figure shows the evolution of the $(r, z)$ beam distribution (in the steadystate regime) for two cases of focusing-electrode voltages $V_{f}$ while maintaining the extraction anode potential to $V_{e}=5 \mathrm{kV}$ thereby exemplifying the effect of the focusing anode voltage.

To further explore the effect of the focusing electrode on the beam formation we present in Fig. 6 the evolution of the rms beam size recorded $6 \mathrm{~mm}$ downstream from the cathode along with the number of macroparticles recorded at the cathode surface and $2 \mathrm{~mm}$ away. For potential $V_{f} \simeq 24 \mathrm{kV}$ the beam reaches a focus at $\mathrm{z}=6 \mathrm{~mm}$. Likewise, we note that the overall transmission (defined as the ratio of current recorded at $\mathrm{z}=2 \mathrm{~mm}$ to the current produced from the cathode) is in excess of $80 \%$; see Fig. 6(bottom) which gives confidence on the overall geometry of the system. However, we note that the current reaches its peak value at $V_{f} \simeq 18 \mathrm{kV}$ corresponding to a collimated beam as seen in Fig. 6(top). These peculiarities come from the interplay between the axial and radial components of the electric field as $V_{f}$ is varied.
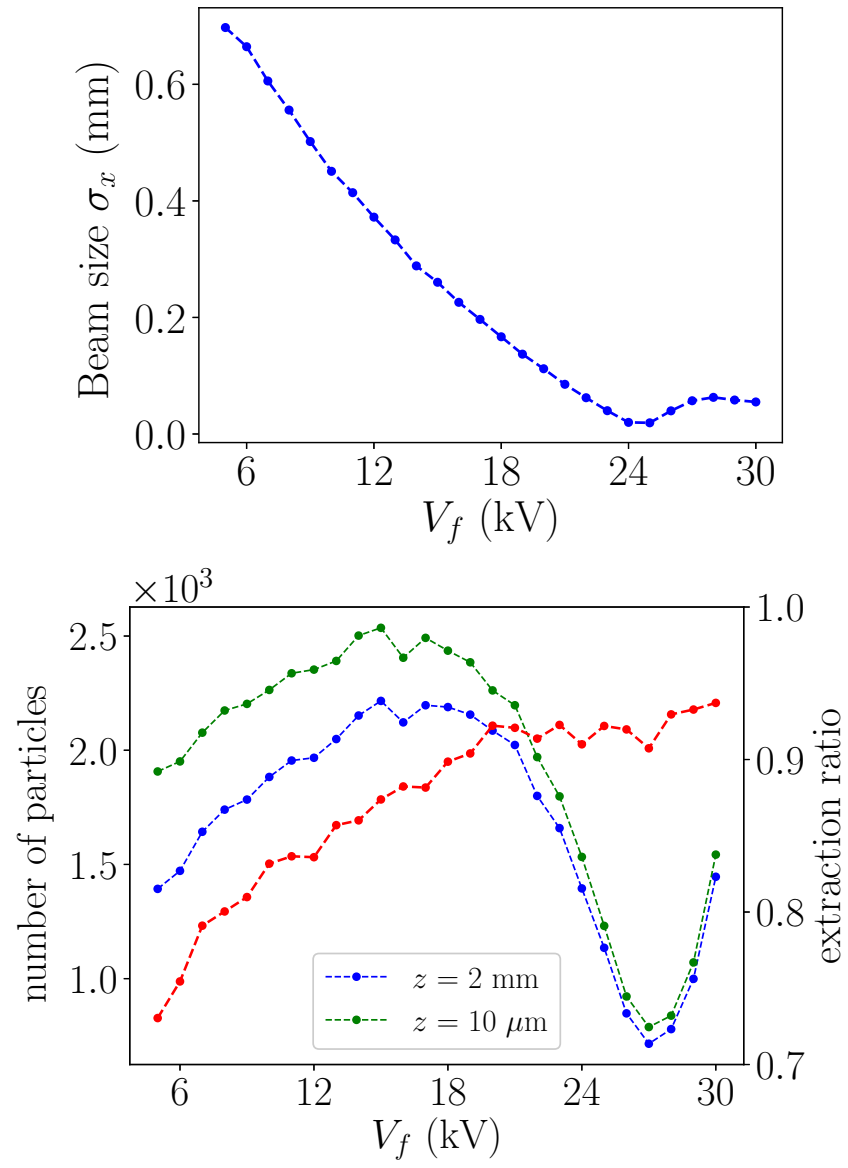

Fig. 6. Evolution of rms beam size $\sigma_{x}$ recorded at $\mathrm{z}=6 \mathrm{~mm}$ (top) and number of particles recorded at at $\mathrm{z}=10 \mu \mathrm{m}$ (green), $2 \mathrm{~mm}$ (blue) (bottom). The lower plot also show the transmission coefficient (red, see text for details). The simulation are recorded at $t=400 \mathrm{ps}$. The first (etraction) anode is at a fixed potential of $5 \mathrm{kV}$ while the focusing electrode is varied together with the screen located on the upper-left boundary of the computational domain. The

\section{Field Enhancement Study}

We now turn to explore the enhancement factor for silicon nanocone FE to be used in our experiment. The FEA consists of a two-dimensional array of nanocone FE tips diagrammed in Fig. 7(top). The field-enhancement factor was investigated with WARP for various nanocone spacing and four bases; see Fig. 7(bottom). For this investigation a small computational domain was used together with a multigrid mesh-refinements electrostatic solver; see detailed in Ref. [10]. As summarized 
in Fig. 7, for a given nanocone height and tip curvature, the field-enhancement factor increases with the nanocone base angle. Likewise, field enhancement asymptotically converges to the single-tip value for large values of the spacing $\left(a_{1}\right)$. Whereas as $a_{1}$ decreases the $\beta_{e}$ decreases owing to the shielding.
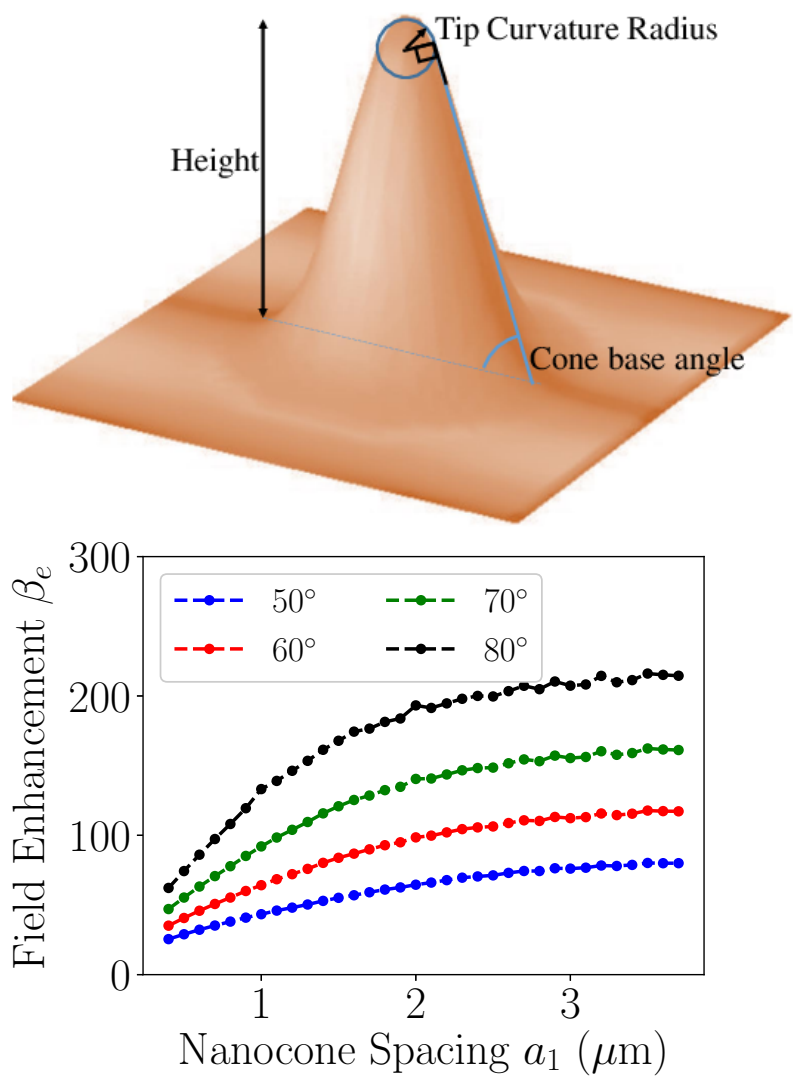

Fig. 7. Nano-cone structure model model used for the field-enhancement investigation and $\beta_{e}$ as a function of spacing between nano-cone structures packed as an array on a cathode for different nano-cone base angles. The cone height and tip curvature radius are fixed to 1 micron and $2 \mathrm{~nm}$, respectively.

\section{Diagnostics \& CONTROLS}

The experimental setup is controlled from a LINUX workstation. A picoammeter (KeITHLEY Model 6485) measures the current flowing between the cathode and ground. The pico-ammeter is interfaced through the workstation using a GPIB protocol interface. Likewise, the HV power supplies are also remotely controllable via GPIB. The various equipment is controlled via the PYVISA [11] a Python implementation of the Virtual Instrument Software Architecture (VISA) that enables the control of various instruments independently of the hardware interface (e.g. GPIB, RS232, USB, Ethernet). This configuration enables high-level scripts to perform automatic tasks (e.g. recording of the $I-V$ curves).

In order to characterize the emitted-beam transverse density distribution, a Cerium-doped Lutetium-yttrium oxyorthosilicate, (LYSO:Ce) scintillator disk of 25-mm diameter and 0.5$\mathrm{mm}$ thick with both sides polished (from Epic-Crystal) is employed. A 5-nm thick Nickel layer was deposited on the screen side facing the cathode. The thickness of the layer is selected such that it is less than the penetration depth of 5$\mathrm{keV}$ electrons. The LYSO:Ce scintillator surface is imaged on a 2.3-mega-pixel CCD camera (model BLACKFLY U3-2356M by FLIR) thereby allowing for the distribution to be recorded. The image acquisition is performed with the AVARIS, a vision library for genicam-based cameras [12]. The images are further analyzed with the IMAGETOOL software [13].

\section{ACKNOWLEDGMENTS}

We are grateful to Drs. D. Grote and J.-L Vay of BerkeleyLab for their help with the WARP program. This work was supported by the US Department of Energy (DOE) contract \# DE-SC0018367, and the National Science Foundation (NSF) Grant \# PHY-1535401 to Northern Illinois University.

\section{REFERENCES}

[1] R. H. Fowler and L. Nordheim, "Electron emission in intense electric fields," Proc. Royal. Soc. London A, vol. 119, no. 781, pp. 173-181, 1928.

[2] L. W. Nordheim, Proc. Royal. Soc. London A, vol. 121, no. 788, p. 626 , 1928.

[3] J. D. Jarvis, H. L. Andrews, B. Ivanov, N. d. J. C. L. Stewar, E. C. Heeres, W.-P. Kang, Y.-M. Wong, J. L. Davidson, and C. A. Brau, J. Appl. Phys., vol. 108, p. 094322, 2010.

[4] K. B. K. Teo, E. Minoux, L. Hudanski, F. Peauger, J.-P. Schnell, L. Gangloff, P. Legagneux, D. Dieumegard, G. A. J. Amaratunga, and W. I. Milne, Nature, vol. 437, p. 968, 2005.

[5] W. S. Graves, F. X. Kärtner, D. E. Moncton, and P. Piot, Phys. Rev. Lett., vol. 108, p. 263904, 2012.

[6] A. Lueangaramwong, D. Mihalcea, G. Andonian, and P. Piot, "Numerical simulations of early-stage dynamics of electron bunches emitted from plasmonic photocathodes," Nucl Instrum Methods Phys Res A, vol. 865, pp. $119-124,2017$

[7] J. Hoffrogge, J. Stein, M. Kruger, M. Forster, J. Hammer, D. Ehberger, P. Baum, and P. Hommelhoff, "Tip-based source of femtosecond electron pulses at 30kev," J. App. Phys., vol. 115, p. 094506, 2014.

[8] W. Zhan, S. Bernal, H. Li, T. Godlove, R. A. Kishek, P. G. O'Shea, M. Reiser, V. Yun, , and M. Venturini, "Design and field measurements of printed-circuit quadrupoles and dipoles," Phys. Rev. ST Accel. Beams, vol. 3, p. 122401, 2000.

[9] A. Friedman, R. H. Cohen, D. P. Grote, S. M. Lund, W. M. Sharp, J. L. Vay, I. Haber, and R. A. Kishek, "Computational methods in the warp code framework for kinetic simulations of particle beams and plasmas," IEEE Trans. Plasma Sci., vol. 42, no. 5, pp. 1321-1334, May 2014.

[10] A. Lueangaramwong, C. Buzzard, R. Divan, V. Korampally, and P. Piot, "Experimental development of low-emittance field-emission electron sources," AIP Conference Proceedings, vol. 1812, no. 1, p. 080009, 2017. [Online]. Available: https://aip.scitation.org/doi/abs/10.1063/1.4975895

[11] "Pyvisa: A python package with bindings to the "virtual instrument software architecture" visa library," https://pyvisa.readthedocs.io, 20002018.

[12] E. Pacaud, "Avaris: A vision library for genicam based cameras," https://github.com/AravisProject/aravis, 2016-2018.

[13] A. Halavanau and P. Piot, "Imagetool: a python-based image analysis," https://github.com/NIUaard/ImageTool, 2015-2018. 